\title{
Gianenrico Bernasconi, Susanne Thürigen (dir.), Material Histories of Time. Objects and Practices, $14^{\text {th }}-19^{\text {th }}$ Centuries
}

Berlin-Boston, De Gruyter, 2020

\section{Louis Georges}

\section{(2) OpenEdition}

\section{Journals}

Édition électronique

URL : https://journals.openedition.org/artefact/10614

DOI : $10.4000 /$ artefact.10614

ISSN : 2606-9245

\section{Éditeur :}

Association Artefact. Techniques histoire et sciences humaines, Presses universitaires du Midi

Édition imprimée

Date de publication : 7 octobre 2021

Pagination : 479-486

ISBN : 978-2-8107-0755-3

ISSN : 2273-0753

\section{Référence électronique}

Louis Georges, «Gianenrico Bernasconi, Susanne Thürigen (dir.), Material Histories of Time. Objects and Practices, $14^{\text {th }}-19^{\text {th }}$ Centuries », Artefact [En ligne], 14 | 2021, mis en ligne le 26 août 2021, consulté le 03 septembre 2021. URL : http://journals.openedition.org/artefact/10614 ; DOI : https://doi.org/10.4000/ artefact. 10614

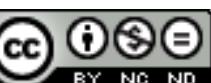

Artefact, Techniques, histoire et sciences humaines est mise à disposition selon les termes de la Licence Creative Commons Attribution - Pas d'Utilisation Commerciale - Pas de Modification 4.0 International. 


\section{Gianenrico Bernasconi, Susanne Thürigen (dir.), Material Histories of Time. Objects and Practices, $14^{\text {th }}-19^{\text {th }}$ Centuries}

Berlin-Boston, De Gruyter, 2020, 226 pages

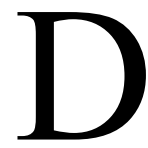

epuis plusieurs années, la multiplication des travaux sur l'histoire de la mesure du temps horaire traduit la volonté de renouveler et de structurer un champ longtemps isolé. Souvent invoquée comme généralité mais peu entreprise pour elle-même, l'étude des relations entre normes horaires, garde-temps, et groupes sociaux a en effet davantage inspiré en effet davantage les entreprises individuelles que les mouvements de groupe. Une raison de cet embarras est, sans nul doute, la polarisation de ce champ entre des historiographies hermétiques, sinon contradictoires : l'histoire économique du temps capitaliste, l'histoire de l'art horloger, les monographies techniques liées à l'antiquariat, plus récemment la socio-histoire des rythmes. Nourrir un dialogue entre ces approches, ouvrir de nouvelles voies méthodologiques, construire une réflexion commune, tels sont les buts que se donne Material histories of time. Objects and practices, $14^{\text {th }}-19^{\text {th }}$ centuries, ouvrage collectif dirigé par Gianenrico Bernasconi et Susanne Thürigen et réunissant 14 contributions, en anglais et en français, à l'histoire de la mesure du temps, de la fin du Moyen Âge à celle du xix ${ }^{e}$ siècle. En intégrant, au prix d'un travail éditorial particulièrement soigné, la nouvelle collection dirigée par Philippe Cordez aux éditions De Gruyter, "Object Studies in Art History ", ces actes d'un colloque tenu en en novembre 2017 au Musée International d'Horlogerie de la Chaux-de-Fonds, a gagné une cohérence épistémologique et stimulante réflexion collective sur les rapports des garde-temps, des pratiques de temporalisation, et des acteurs sociaux.

Inspirée par l'approche praxéologique développée dans l'historiographie allemande ${ }^{1}$, et prolongeant des réflexions sur le rôle du corps et des gestes dans l'histoire des techniques ${ }^{2}$, l'unité donnée à l'ouvrage par ses deux directeurs est fondée sur une ontologie de l'objet technique comme dispositif socialisé, indissociable de pratiques qui lui donnent sens. Montres

1. Haasis et Rieske, 2015.

2. Bernasconi, 2016 ; Thürigen, 2018. 
et horloges ne sont pas en société les prouesses mécaniques que les horlogers célèbrent ; elles sont des éléments dans un ensemble de matérialités et de manipulations qui produisent l'orientation temporelle d'un acteur. L'objet inclut des "programmes d'action " qui suggèrent les gestes des utilisateurs, mais les pratiques effectives "braconnées " impriment en retour leurs marques sur lui. Les instruments conservés deviennent, par les traces matérielles de leur manipulation rituelle, les vestiges de ce qu'aucun texte ne décrit, et deviennent des documents susceptibles de lectures et d'interprétations.

Ce cadre posé, les contributions s'organisent selon des connivences thématiques, quoiqu'en respectant un développement chronologique sommaire. Un premier ensemble considère les liens des horloges médiévales et modernes aux pratiques politiques qui en font usage. Cette culture matérielle rare s'inscrit en effet dans une expression du pouvoir incarné, et suscite des gestes tenant du symbole plus que de l'orientation temporelle. Étudiant les horloges astronomiques encore édifiées tard dans l'époque moderne, Günther Oestmann ${ }^{3}$ formule ainsi un rappel fondamental contre le déterminisme technique : la visualisation plus fréquente de l'heure par le cadran n'implique aucunement que celle-ci soit lue et utilisée en tant qu'information. Les grandes horloges n'ont pas tant pour but d'informer sur le temps horaire que d'incarnert une certaine représentation du cosmos, en particulier dans le contexte des conflits religieux. La vertu d'une approche par la« lecture » des objets à l'image d'une source textuelle, qu'il s'agisse d'une fontaine hydraulique tardo-médiévale ${ }^{4}$ ou d'une horloge de table de $1586^{5}$ montre la vertu d'une telle approche permet de ramener les garde-temps au contexte socio-politique de leur production plutôt qu'à un jugement technique formulé à partir de critères postérieurs.

Trois contributions se saisissent ensuite d'une thèse longtemps influente : celle d'une "révolution horlogère " au XVIII ${ }^{\mathrm{e}}$ siècle. Ce schéma topique, postulant un tournant socio-économique lié à la régularité de l'horlogerie post-huygensienne, fait l'objet d'une triple critique, touchant la représentation picturale des instruments, dont la valeur change plus que la fréquence ${ }^{6}$,

\footnotetext{
3. Günther Oestmann, 2020.

4. Cordez, 2020.

5. Pérez-Álvarez, 2020.

6. Dohrn-van Rossum, 2020.
} 
leur diffusion, plus complexe que la seule vente à des acteurs passifs ${ }^{7}$, l'intériorisation d'une " conscience temporelle " moderne. Gerrit Verhoeven ${ }^{8}$, constatant le décalage de la disponibilité accrue de l'heure mécanique, avec la stabilité de formulations horaires anciennes dans les dépositions enregistrées au Old Bailey au XVIII ${ }^{\mathrm{e}}$ siècle, nuance cette dernière idée, ainsi que l'étude célèbre de Hans-Joachim Voth sur les mêmes sources ${ }^{9}$. Une archéologie des gestes dissipe ce paradoxe : il faut, pour lire l'heure, s'arrêter ou arrêter son cheval, sortir l'objet, le retirer de son étui, relever le cache... Le geste dispose donc d'une certaine épaisseur, expose un objet prisé aux voleurs, et 'est donc pas aussi fréquent qu'à l'époque contemporaine. La montre n'est ainsi pas toujours un garde-temps : la possession n'induit pas une utilisation, moins encore une intériorisation. Les pratiques sont ici un moyen de mettre en cause les récits déterministes ; le schéma logique d'une diffusion tirée par l'offre technique étant démenti par l'étude empirique.

Une autre cohérence thématique concerne la dimension temporelle des mobilités au XVIII ${ }^{\mathrm{e}}$ siècle. L'originalité de l'ensemble est de mettre en lien l'émergence d'une conception métrologique des phénomènes ${ }^{10}$ à la fin de l'époque moderne, avec le développement du voyage et du transport de ligne, fondé sur la disponibilité d'une information horaire statique (les tables horaires), et dynamique (l'horlogerie de voyage $)^{11}$, qui rend sensible des conflits de synchronisation et de référentialité, devenus enjeux culturels à la faveur de la diffusion du modèle grand-touriste ${ }^{12}$. La contribution de Nicolas Verdier ${ }^{13}$, particulièrement dense, examine la séparation progressive de l'espace et du temps comme échelle des représentations cartographiques. Au XVII ${ }^{\mathrm{e}}$ siècle, la notion de "distance " concerne indifféremment une distance-terrain ou une distance-temps. Les pratiques de voyage séparent progressivement les deux notions : pour décrire des itinéraires viaires, l'éloignement théorique n'est pas si important que le temps de trajet effectif. D'où les efforts, au XVIII ${ }^{\mathrm{e}}$ siècle, pour produire des tables horaires empiriques à l'aide d'un compteur fixé à l'essieu des

\footnotetext{
7. Dequidt, 2020.

8. Verhoeven 2020.

9. Voth, 2000.

10. Frängsmyr, Heilbron et Rider (dir.), 1990.

11. Herr-Laporte, 2020.

12. Besson, 2020.

13. Verdier, 2020.
} 
voitures, l'odomètre. Dans cette séparation notionnelle se joue l'autonomisation d'un savoir du temps, qui accompagne sa mesure et sa précision.

La temporalisation des activités sociales, et sa médiatisation progressive par les dispositifs horlogers fait l'objet d'un autre ensemble. La construction d'une " mesure » du temps, au sens d'un ensemble de données permettant la comparabilité des durées, est ici historicisée, par la mise en place d'une discipline scolaire organisée par le découpage horaire d'une discipline scolaire collective ${ }^{14}$, ou, de manière originale, par les livres de recettes des XVII et XVIII ${ }^{\mathrm{e}}$ siècles qui utilisent l'heure d'horloge comme l'instrument d'une formalisation des pratiques par l'écrit ${ }^{15}$. On retiendra le stimulant travail de Marco Storni ${ }^{16}$ qui considère les pratiques chronométriques du dispositif expérimental de Lavoisier. Au sein des débats sur la distinction des objets techniques et scientifiques, Storni avance l'idée d'un gradient entre les objets spécifiques au cadre expérimental, et les objets ordinaires qui participent de la scientificité sans, souvent, être évoqués, à l'image de la montre chez Lavoisier. Le rôle épistémique de cette petite culture matérielle amène l'auteur à théoriser les contours des "savoirs évidents " (taken-for-granted knowledge), qui ne sont ni discutés, ni contestés par les acteurs, mais qui servent de critères à la validation ou au rejet du savoir expérimental. On trouvera dans cette théorisation un cadre pertinent pour penser l'émergence de pratiques de mesure, constitutives d'une nouvelle appréhension du réel fondée sur une médiation technique devenue évidente.

Un dernier ensemble s'intéresse aux pratiques méconnues de la profession horlogère aux XVIII ${ }^{e}$ et XIX ${ }^{e}$ siècles. Quand l'historiographie s'est concentrée sur les grandes figures et sur l'innovation technique sous un mode laudatif, les revenus des horlogers proviennent surtout des reventes, des réparations, et de la maintenance locale, comme le montre le livre de compte d'un modeste horloger de Nouvelle-Angleterre au XIX ${ }^{\mathrm{e}}$ siècle $^{17}$. L'histoire même $s$ 'inscrit dans ces pratiques professionnelles : les pratiques de falsifications techniques et narratives inventent des histoires aux horloges pour les besoins de leurs reventes, dans le cadre d'un marché antiquaire qui donne

14. Pruneri, 2020.

15. Bernasconi, 2020.

16. Storni, 2020.

17. McCrossen, 2020. 
une valeur économique aux récits historiques ${ }^{18}$. Les petits récits historiques accompagnant les pratiques commerciales participent, en s'additionnant, à la construction d'une histoire déterministe de l'horlogerie, fondée sur la valeur technique de chefs d'œuvre consacrés par le regard téléologique du marché.

Material Histories of Time est une contribution majeure à l'histoire de la mesure du temps, à celle des savoirs d'action et des cultures techniques de l'ordinaire. On saluera le grand soin apporté à l'édition et à la riche iconographie, qui complète habilement l'approche par les objets. Profitant du renouveau d'un sujet souvent lié aux débats économiques des années 1970 et 1980, ce recueil est à la fois un condensé des derniers apports historiographiques, et un catalyseur pour la recherche future. Par son souci du travail de la source, l'approche praxéologique autorise un intéressant retournement épistémologique : il ne s'agit plus de cerner les vagues contours d'une " conscience temporelle " ou d'un insaisissable mystère métaphysique, mais de ramener la différenciation des moments de la journée à des pratiques incarnées, ancrée dans une matérialité accessible et lisible, et exprimée dans des gestes sociaux. À ce titre, l'entreprise, mêlant des historiens éminents à de jeunes chercheurs, doit avant tout être louée comme une enthousiaste promesse épistémologique participant de la construction d'un champ historiographique.

Parmi les voies suggérées, on retiendra le souci de dénaturaliser la notion, polysémique à l'extrême et souvent porteuse d'une abstraction suspecte, de " temps ». Plus qu'une entité ontologique ou une réalité statistique, c'est l'action de mesurer, de normer, et de réduire le mouvement en données manipulables, qui forme le sujet de ces études. Le temps est ainsi entendu comme " temporalisation " : regarder sa montre, la régler, consulter une méridienne ou des tables horaires, sont des réalités sociales identifiables et susceptibles d'études. L'effet de cette perspective est double. D'une part, l'approche praxéologique introduit la notion de "pratique temporelle " incluant, parmi les "savoirs pratiques ", les gestes entourant l'information horaire, son acquisition, sa transmission, ou son utilisation. D'autre part, lire les gestes et les objets pour eux-mêmes permet de mettre à distance les impositions téléologiques et de revenir à l'étrangeté des temporalisations

18. Graf, 2020. 
passées. Les critères contemporains de rationalité étant le produit du mouvement historique de formalisation des mesures à partir du XvIII ${ }^{\mathrm{e}}$ siècle, une telle recherche revient à porter un regard archéologique sur les contours sociaux de la perception et de l'identification du réel.

Sans doute pourra-t-on souligner la détermination des terrains de recherche, essentiellement européens, essentiellement techniciens, essentiellement tardo-modernes, et en cela paradoxalement proches de la littérature de la "révolution horlogère ». On regrettera par exemple que toutes les contributions concernent l'information horaire privée : l'approche par les objets favorise l'horlogerie de table et de corps plutôt que celle d'édifice, pourtant plus importante pour les acteurs modernes. Mais ces choix apparaissent moins comme des limites que comme des invitations : la fertilité des approches formulées et réunies dans Material Histories of Time, ouvre de nouveau un chantier longtemps à l'arrêt, l'histoire sociale des temporalités.

Louis GeORGES

Université Paris-Nanterre, Centre d'histoire des sociétés médiévales et modernes (MéMo)

\section{Bibliographie}

Bernasconi Gianenrico, "Temps et cuisine, XVII ${ }^{\mathrm{e}} \mathrm{XVIII}{ }^{\mathrm{e}}$ siècles. Remarques sur les pratiques de transformation alimentaire ", dans Bernasconi Gianenrico et Thürigen Susanne (dir.), Material Histories of Time. Objects and Practices, $14^{\text {th }}-19^{\text {th }}$ Centuries, De Gruyter, Berlin-Boston, 2020, p. 173-187

Bernasconi Gianenrico, "L'objet comme document. Culture matérielle et cultures techniques ", Artefact. Techniques, histoire et sciences humaines, 4-4, octobre 2016, p. 31-47.

Bernasconi Gianenrico, Objets portatifs au siècle des Lumières, Éditions du Comité des travaux historiques et scientifiques, Paris, 2015.

Bernasconi Gianenrico et Thürigen Susanne (dir.), Material Histories of Time. Objects and Practices, $14^{\text {th }}-19^{\text {th }}$ Centuries, De Gruyter, Berlin-Boston, 2020

Besson Grégoire, "Les voyageurs et leurs montres en Europe à la fin du $\mathrm{XVIII}^{\mathrm{e}}$ siècle. Entre précision technique et flou temporel ", dans Bernasconi Gianenrico et ThürIgen Susanne (dir.), Material Histories of Time. Objects and Practices, $14^{\text {th }}-19^{\text {th }}$ Centuries, Berlin-Boston, De Gruyter, 2020, p. 143-155 
Cordez Philippe, "Musique et Jouvence au royaume de France. Le Roman de Fauvel et la fontaine de Cleveland (Paris, vers 1320) ", dans Bernasconi Gianenrico et Thürigen Susanne (dir.), Material Histories of Time. Objects and Practices, $14^{\text {th }}-19^{\text {th }}$ Centuries, De Gruyter, 2020, Berlin-Boston, p. 17-39

Dequidt Marie-Agnès, «De l'acquisition au réglage. L'horlogerie parisienne du XVIII ${ }^{\mathrm{e}}$ siècle et ses usages ", dans Bernasconi Gianenrico et Thürigen Susanne (dir.), Material Histories of Time. Objects and Practices, $14^{\text {th }}-19^{\text {th }}$ Centuries, Berlin-Boston, De Gruyter, 2020, p. 89-102

Dohrn-van Rossum Gerhard, "Clocks, clock time, and time consciousness in the visual arts. William Hogarth's modern moral subjects ", dans BERNASCONI Gianenrico et Thürigen Susanne (dir.), Material Histories of Time. Objects and Practices, $14^{\text {th }}-19^{\text {th }}$ Centuries, De Gruyter, Berlin-Boston, 2020, p. 71-88

Frängsmyr Tore, Heilbron J. L. et Rider Robin E., The Quantifying Spirit in the 18th Century, University of California Press, Berkeley, 1990.

FREIST Dagmar, Diskurse-Körper-Artefakte : Historische Praxeologie in der Frühneuzeitforschung, Aufl., München, transcript, 2015.

Graf Johannes, "The First black forest clock. How a fake impacted our notion of clock history ", dans Bernasconi Gianenrico et Thürigen Susanne (dir.), Material Histories of Time. Objects and Practices, $14^{\text {th }}-19^{\text {th }}$ Centuries, De Gruyter, Berkeley, 2020, p. 211-224

HaAsis Lucas et Rieske Constantin, Historische Praxeologie. Dimensionen vergangenen Handelns, Schöningh, Paderborn, 2015.

Herr-Laporte Catherine, "Les montres de voyage. Désignations, techniques et usages (1700-1830), dans Bernasconi Gianenrico et Thürigen Susanne (dir.), Material Histories of Time. Objects and Practices, $14^{\text {th }}-19^{\text {th }}$ Centuries, De Gruyter, Berlin-Boston, 2020, p. 129-144.

McCrossen Alexis, "Broken watches, sources and methods for the study of time consciousness ", dans Bernasconi Gianenrico et Thürigen Susanne (dir.), Material Histories of Time. Objects and Practices, $14^{\text {th }}-19^{\text {th }}$ Centuries, De Gruyter, Berlin-Boston, 2020, p. 201-210

Oestmann Günther, "Designing a model of the cosmos. Theoretical and visual considerations concerning the construction of astronomical clocks through early modern times ", dans Bernasconi Gianenrico et THürigen Susanne (dir.), Material Histories of Time. Objects and Practices, $14^{\text {th }}-19^{\text {th }}$ Centuries, De Gruyter, Berlin-Boston, 2020, p. 41-54

PÉrez-Állvarez Victor, "The $16^{\text {th }}$-century astronomical clock of the National Maritime Museum of Greenwich. Cultural and sensorial analysis ", dans Bernasconi Gianenrico et Thürigen Susanne (dir.), Material Histories of Time. Objects and Practices, $14^{\text {th }}-19^{\text {th }}$ Centuries, De Gruyter, Berlin-Boston, 2020, p. 55-70 
Comptes rendus de lecture

Pruneri Fabio, "Time management at school from the late middle ages to the industrial age. A Few cases in point ", dans Bernasconi Gianenrico et Thürigen Susanne (dir.), Material Histories of Time. Objects and Practices, $14^{\text {th }}-19^{\text {th }}$ Centuries, De Gruyter, Berlin-Boston, 2020, p. 157-172

STORNi Marco, "Clocks and timekeeping in Lavoisier's experiments on animal respiration ", dans Bernasconi Gianenrico et Thürigen Susanne (dir.), Material Histories of Time. Objects and Practices, $14^{\text {th }}-19^{\text {th }}$ Centuries, De Gruyter, BerlinBoston, 2020, p 187-200

Valleriani Matteo, The Structures of Practical Knowledge, New York, Springer, 2017.

Verdier Nicolas, "La montre et l'odomètre. À propos de l'Itinéraire des routes les plus fréquentées de Louis Dutens (1775) ", dans Bernasconi Gianenrico et Thürigen Susanne (dir.), Material Histories of Time. Objects and Practices, $14^{\text {th }}-19^{\text {th }}$ Centuries, De Gruyter, Berlin-Boston, 2020, p. 117-127

Verhoeven Gerrit, "Time technologies. Londoners and their timepieces (17751825) ", dans Bernasconi Gianenrico et Thürigen Susanne (dir.), Material Histories of Time. Objects and Practices, $14^{\text {th }}-19^{\text {th }}$ Centuries, De Gruyter, BerlinBoston, 2020, p. 103-115

Vотн Hans-Joachim, Time and Work in England 1750-1830, Clarendon Press, Oxford, 2000. 